ISSN 1112-9867

Available online at

http://www.jfas.info

\title{
NUMERICAL INVESTIGATION OF NATURAL CONVECTION HEAT TRANSFER OF NANO-FLUIDS IN A MICRO-CHANNEL
}

\author{
F. Rouzbahani ${ }^{1, *}$ and R. Dehnavi ${ }^{2}$ \\ ${ }^{1}$ Department of Mechanical Engineering, Hamedan branch, Islamic Azad University, Hamedan, Iran \\ ${ }^{2}$ Department of Mechanical Engineering, Broojerd branch, Islamic Azad University, Broojerd, Iran
}

Published online: 05 June 2016

\begin{abstract}
An enhanced cell-centered finite-volume procedure has been presented for solving the natural convection of the laminar $\mathrm{Al}_{2} \mathrm{O}_{3}$ /Water nanofluid flow in a $\Gamma$ shaped micro-channel in the slip flow region, including the effects of velocity slip and temperature jump at the wall, which are the main characteristics of flow in the slip flow region. In provided FORTRAN code, an explicit fourth-order Runge-Kutta integration algorithm has been applied to find the steady state solutions. Also an artificial compressibility technique has been to couple the continuity to the momentum equations. The Grashof numbers from $10^{2}$ to $10^{5}$ have been considered. Results show that decreasing the mean diameters of nanoparticles, increases the Nusselt number. Increasing the mean nanoparticle volume fraction increases the Nusselt number. Also by increasing the Knudsen number, the Nusselt number decreases.
\end{abstract}

Keywords: velocity slip; temperature jump; Knudsen number; nanoparticle.

Author Correspondence, e-mail: f.rouzbahani@iauh.ac.ir

doi: http://dx.doi.org/10.4314/jfas.8vi2s.60

\section{Introduction}

The fluid transport in the micro-channels plays a vital role in a wide variety of contemporary engineering applications, involving micro-scale devices such as micro-pumps, micro-valves and 
micro-sensors. Particularly, the application of micro-channels in electronic cooling is becoming tremendously important due to the increasingly high-flux heat generation from high-speed microprocessors. On the other hand Advancement in the electronics industry led to the development of micro-scale heat transfer devices which offered high heat transfer coefficient in a compact size. Nevertheless, the heat transfer characteristics were limited by the heat transfer fluids that were used, low thermal conductivity of the conventional heat transfer fluids such as water, oil, and ethylene glycol mixture is a serious limitation in improving the performance and compactness of these engineering equipments. To overcome this disadvantage, there is a strong motivation to develop advanced heat transfer fluids with substantially higher conductivity. One way is adding small solid particles in the fluid. For example, using $\mathrm{Al}_{2} \mathrm{O}_{3}$ particles having a mean diameter of $13 \mathrm{~nm}$ at $4.3 \%$ volume fraction increased the thermal conductivity of water under stationary conditions by $30 \%$ [1]. An increasing number of experimental investigations have demonstrated that nano-fluids can dramatically increase the effective thermal conductivity of the base fluid [2-4].

The Knudsen number relates the molecular mean free path to a characteristic dimension of the duct. Knudsen number is very small for continuum flows. However, for micro-scale flows where the mean free path becomes comparable with the characteristic dimension of the duct, the Knudsen number may be greater than $10^{-3}$. Micro-channels with characteristic lengths on the order of $100 \mu \mathrm{m}$ would produce flows inside the slip regime with a typical mean free path of approximately $100 \mathrm{~nm}$ at standard conditions. The slip flow regime to be studied here is classified as $10^{-3} \leq \mathrm{Kn} \leq 10^{-1}$. It is well established that for this range of Knudsen numbers (slip-flow regime), the standard Navier-Stokes and energy equations can still be used with modifications to the boundary conditions allowing for velocity-slip and temperature-jump at the walls.

Lelea and Nisulescu investigated the numerical modeling of the conjugate heat transfer and fluid flow of $\mathrm{Al}_{2} \mathrm{O}_{3}$ /water nanofluid through the micro-tube. The water based $\mathrm{Al}_{2} \mathrm{O}_{3}$ nanofluid was considered with various volume concentrations of $\mathrm{Al}_{2} \mathrm{O}_{3}$ particles $1,4,6,9 \%$ and two diameters of the particles $\mathrm{Dp}=10 \mathrm{~nm}$ and $47 \mathrm{~nm}$, the analysis was performed on the results for local heat transfer coefficient [5]. Ch.Ch. Choa et al. investigated the natural convection heat transfer characteristics and entropy generation of water-based nanofluids in an enclosure bounded by wavy vertical walls and flat upper and lower surfaces. Their analysis considers three different 
nanofluids, namely $\mathrm{Cu}$-water, $\mathrm{Al}_{2} \mathrm{O}_{3}$-water andTiO $\mathrm{T}_{2}$-water. Their results show that for all values of the Rayleigh number, the mean Nusselt number increases and the total entropy generation reduces as the volume fraction of nanoparticles increases [6]. A. H. Mahmoudia et al. investigated the entropy generation and enhancement of heat transfer in natural convection flow and heat transfer using Copper $(\mathrm{Cu})$-water nanofluid in the presence of a constant magnetic field. The analysis uses a two dimensional trapezoidal enclosure with the left vertical wall and inclined walls kept in a low constant temperature and a heat source with constant heat flux placed on the bottom wall of the enclosure. The governing equations were discretized by the control volume method and solved numerically by SIMPLE algorithm. Their results show that at $\mathrm{Ra}=10^{4}$ and $10^{5}$ the enhancement of the Nusselt number due to presence of nanoparticles increases with the Hartman number, but at higher Rayleigh number, a reduction has been observed [7].Arie et al. carried out a computational optimization analysis on a full-scale manifold-micro-channel heat exchanger[8]. P. FariñasAlvariño et al. studied a numerical investigation of a tubular developing laminar flow of a water/alumina nanofluid under constant heat flux conditions. The nanofluid is modeled as a water/alumina nanoparticles mixture [9]. Ebrahimi et al. performed a numerical simulation on the liquid flow and heat transfer in a rectangular micro-channel with longitudinal vortex generators (LVGs)[10]. Yue et al. simulated a manifold micro-channel heat sink employing nanofluids as heat transfer medium. Their results showed that increasing the volume concentration of nanofluids leads to an increase in Nusselt number and pumping power while entropy generation plummets [11].Hedayati et al. studied the effects of nanoparticle migration and asymmetric heating on mixed convection of $\mathrm{TiO}_{2}$ /water nanofluid inside a vertical microchannel [12]. X. D. Wang analyzed the flow and heat transfer in nanofluid-cooled micro-channel heat sink (MCHS) [13]. S. M. Hosseini Hashemi et al. studied forced convection heat transfer in a porous annular micro-duct. Brinkman extended Darcy equation was used and the porous medium shape parameter, the velocity slip and the temperature jump at the walls are taken into account. Two distinct thermal boundary conditions were analyzed: constant heat flux at the outer cylinder and insulated inner cylinder and vice versa. Results show that for both cases Nusselt number decreases as Knudsen number and annulus aspect ratio increase [14]. Qinbo Hea preparedCu $-\mathrm{H}_{2}$ Onanofluids through two-step method.. The factors influencing transmittance of nanofluids, such as particle The transmittance of nanofluids over solar spectrum (250-2500 nm) was measured by the UV-Vis-NIR spectrophotometer based on integrating sphere principle size, 
mass fraction and optical path were investigated [15]. Massimo Corcione proposed and discussed two empirical correlations for predicting the effective thermal conductivity and dynamic viscosity of nanofluids, based on a high number of experimental data available in the literature. He found that, given the nanoparticle material and the base fluid, the ratio between the thermal conductivities of the nanofluid and the pure base liquid increases as the nanoparticle volume fraction and the temperature are increased, and the nanoparticle diameter is decreased [16]. Zhu et al. studied the laminar flow and heat transfer of /ethylene glycol-water nanofluids in a wavy finned heat sink[17]. The finite volume method of Jameson et al. has proved to be useful as a tool in solving the Euler and Navier-Stokes equations [18-20]. In the last numerical solutions of the heat transfer and fluid flow for enclosures, a code based on the enhanced SIMPLE method [21], almost couples the pressure to the velocity. In this work, an artificial compressibility technique is applied to couple the continuity to the momentum equations. This technique was originally introduced by Chorin with the objective of solving the steady state incompressible Navier-Stokes equations. Chorin transformed the elliptic incompressible equations to a hyperbolic compressible system, which can be solved by standard, time-marching methods. The idea of relaxing the incompressibility constraint by adding an artificial compressibility term has been known for a long time and has been extensively used in finite-volume as well as in finite element approximations of the incompressible Navier-Stokes equations [22-25]. The discretization of the viscous and thermal conduction terms are very simplified using the enhanced scheme similar to flux averaging in the convective terms. This enhanced method has been introduced by Ziapour and Dehnavi [26].

Due to the lack of information about non-square and rectangular enclosure, this study investigates the flow field and heat transfer in a $\Gamma$ shaped micro-channel that is filled with $\mathrm{Al}_{2} \mathrm{O}_{3}$-watre nanofluid. Thermal conductivity and dynamic viscosity of the nanofluid are employed by Xu's model [27] and Jang's model [28], respectively.

\section{RESULTS AND DISCUSSIONS}

The validity of the numerical approach has been assessed by the grid-independence and comparisons with the results in the literature. The present problem for a specific situation has performed for four different grids $(50 \times 50,100 \times 100,150 \times 150$, and $200 \times 200)$ has performed. The 
maximum Nusselt difference between the four listed grids is less than $1.5 \%$. Finally for saving time and maintaining accuracy the $100 \times 100$ grid has been found suitable for the present problem. As already mentioned, a similar study for the nanofluid flow in a $\Gamma$ shaped micro-channel is not available. Therefore, in order to verify the present model, the code for nanofluid inside the ordinary enclosure (not micro-channel) has been run, and obtained Nusselt numbers have beencompared with reference [29], and results are completely matched.

The effects of Grashof number, the properties and the distribution of nanoparticles (mean nanoparticle diameter, $\mathrm{R}$ parameter and nanoparticle volume fraction), and Knudsen number on the fluid flow, isotherms, streamlines and Nusselt number have been investigated.

\subsection{Isotherms}

Effecst of Grashof number and Knudsen number on isotherms

Isotherms for $\mathrm{Gr}=10^{2}$ to $\mathrm{Gr}=10^{5}$ in $\mathrm{dp}=5 \mathrm{~nm}$ have been plotted in figure $(1-\mathrm{a})$ for $\mathrm{Kn}=$ 0.001 , figure (1-b) for $\mathrm{kn}=0.01$ and figure (1-c) for $\mathrm{kn}=0.1$.

As is obvious from the figure (1-a), by increasing Grashof number the distortion of isotherms increases too, it is due to the increasing of buoyancy force and getting stronger convection inside the chamber. By increasing Knudsen number the distortion of isotherms decreases, because as the Knudsen number increases, the temperature jump at the walls increases too. The temperature jump at the walls reduces the heat transfer. 

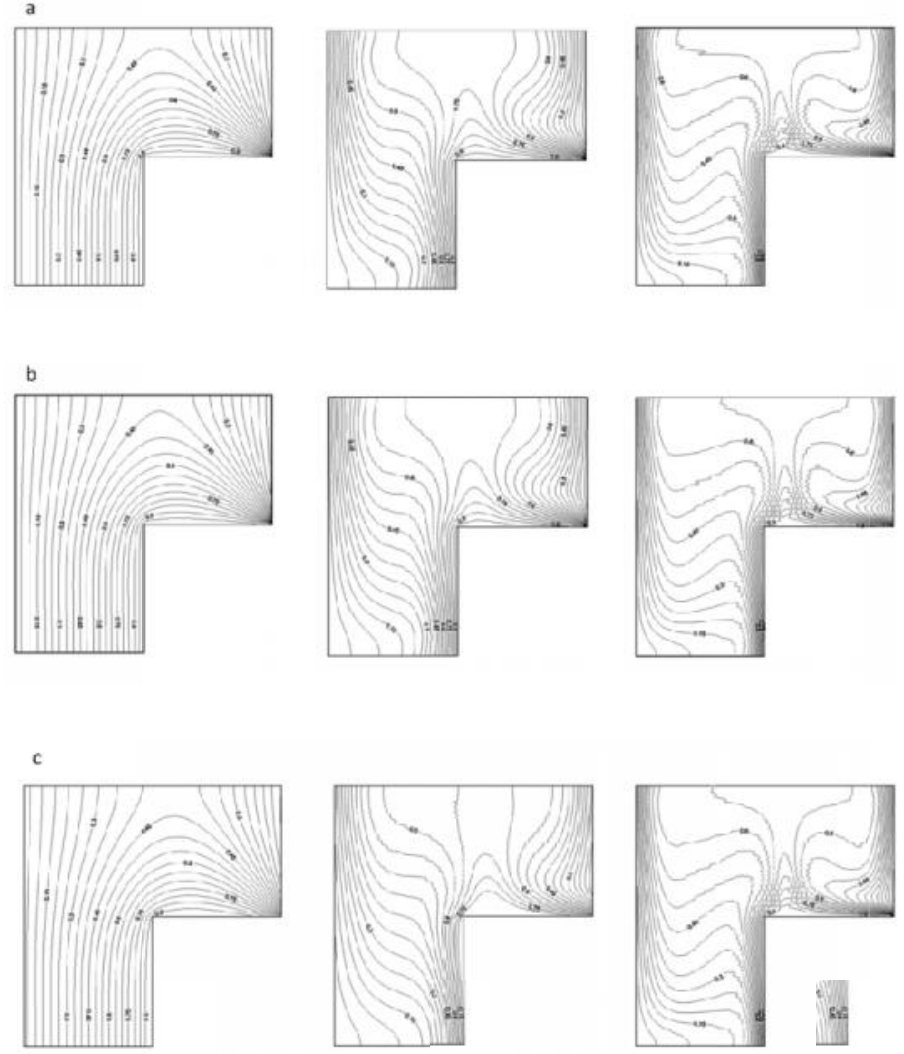

Fig.1. Isotherms forGr $=10^{2}$ (left), $\mathrm{Gr}=10^{4}$ (middle) and $\mathrm{Gr}=10^{4}$ (nght), $(\mathrm{a}: \mathrm{Kn}=0.001$, $\mathrm{b}: \mathrm{Kn}=0.01, \mathrm{c}: \mathrm{Kn}=0.1)$

Effect of nanopartic olume fraction on isotherms

Isotherms for $=10^{2}(1 \mathrm{dp}=5 \mathrm{~nm}, \mathrm{Kn}=0.01, \mathrm{R}=0.002$, in different nanoparticle volume fraction have been plotted in figure (2). The isotherms are not significantly affected by nanoparticle volume fraction. However, by increasing nanoparticle volume fraction the distortion of isotherms increases too. This is related to an increase in the inertia forces as depicted by Eqs. (2) and (3), the equation shows that the inertia forces are amplified by the nanoparticle volume fraction. Therefore, any increase in volume fraction increases the inertia forces and accordingly the temperature gradient and the heat transfer. 

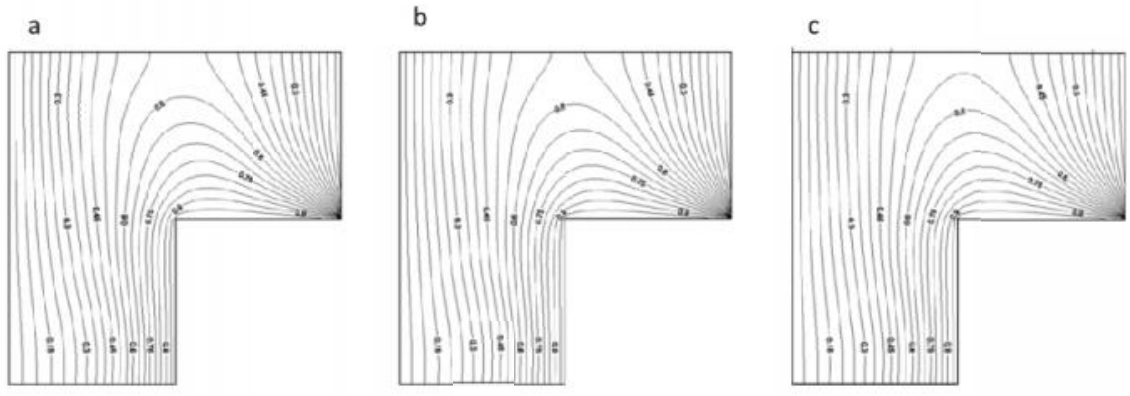

Fig.2. Isotherms in $\mathrm{Gr}=10^{4}(\mathrm{a}: \phi=0.01, \mathrm{~b}: \phi=0.025, \mathrm{c}: \phi=0.05)$

Effects of non-uniform nanoparticle diameter and mean nanoparticle diameter on isotherms

The effect of fractal distributions on the heat transfer is reported in the terms of the ratio of minimum to maximum nanoparticle diameter $R$. $R$ values provides the importance of the nonuniform nanoparticle structures. Figure (3-a) shows isotherms for different R numbers, while $\mathrm{Gr}=10^{4}, \phi=0.05, \mathrm{Kn}=0.01, \mathrm{~d}_{\mathrm{p}}=5 \mathrm{~nm}$. By increasing $\mathrm{R}$ parameter the distortion of isotherms increases too. The phenomenon can be explained based on the Brownian motion theory.
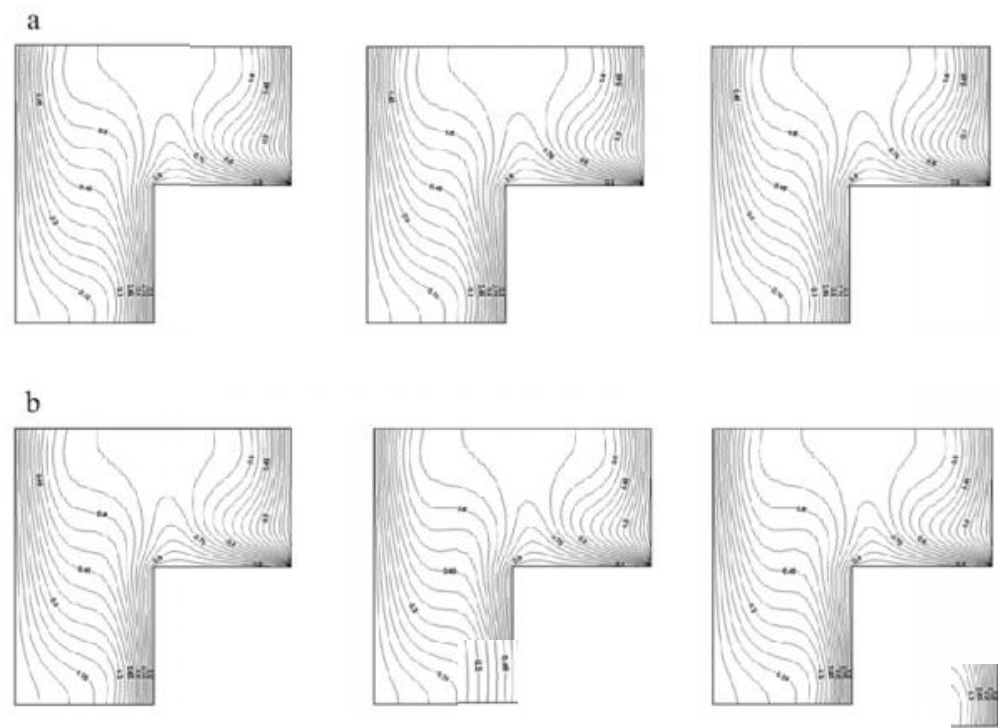

Fig.3. Isotherms in $\mathrm{Gr}=10^{4}$ (a:: different $\mathrm{R}, \mathrm{b}$ : different $\mathrm{d}_{4}$ (a

To study the effect of the mean nanop nar $_{\mathrm{e}}$ ( heter on the heat transfer, we vary the diameter

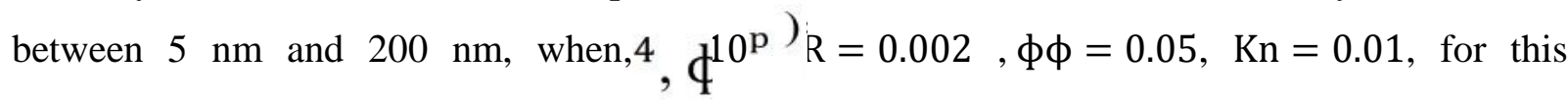
situations isotherms have been plotted in t1gure (3-b). The isotherms are not significantly affected 
by mean nanoparticle diameter. However, by increasing mean nanoparticle diameter the distortion of isotherms decreases. Decreasing the diameters of nanoparticles has qualitatively the same effect increasing $\mathrm{R}$.

\subsection{Stream lines}

Stream lines have been shown in figure (4), the figures have been sketched for Gr $=10^{5}=10^{2}(1$ , $\mathrm{dp}=5 \mathrm{~nm}, \phi \phi=0.05$, in different Knudsen numbers.
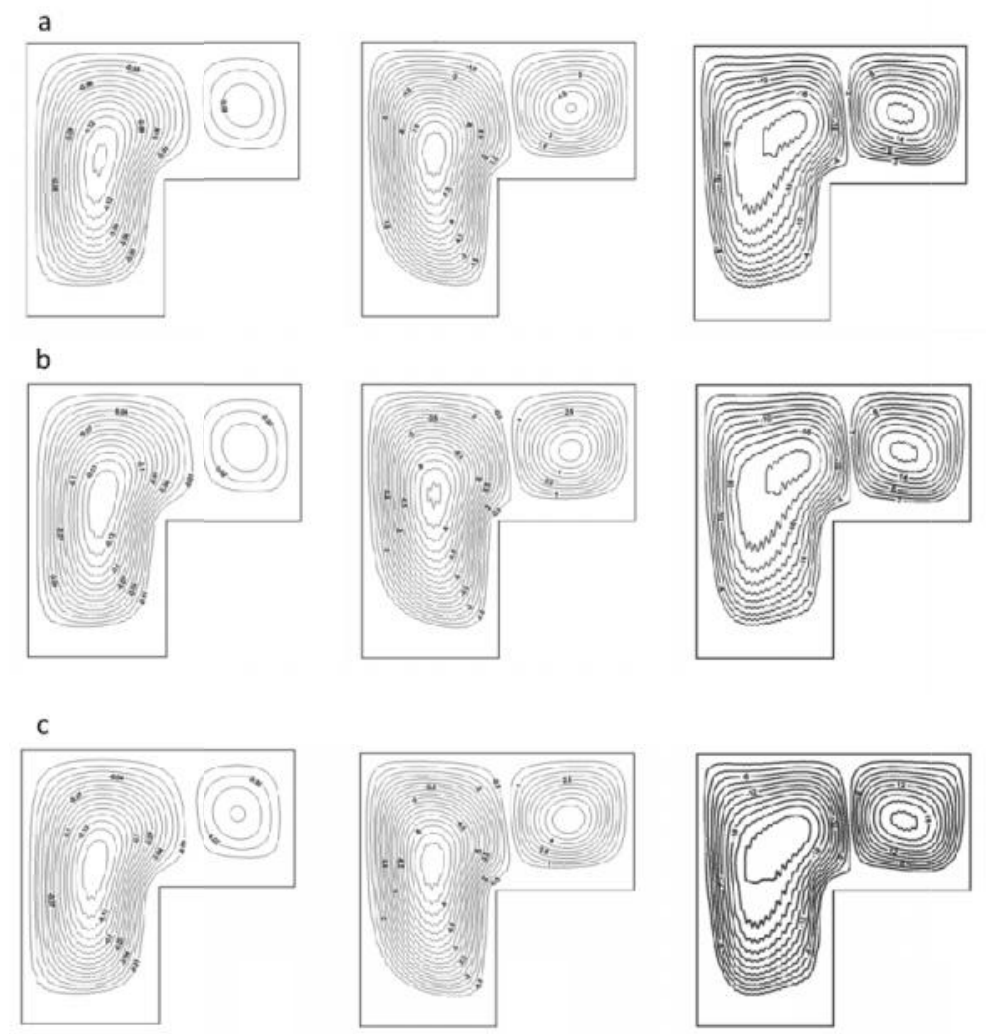

Fig.4. Streamlines forGr $=10^{2}$ (left), $\mathrm{Gr}=10^{4}$ (middle) and $\mathrm{Gr}=10^{4}$ (nght), $(\mathrm{a}: \mathrm{Kn}=0.001$,

$$
\mathrm{b}: \mathrm{Kn}=0.01, \mathrm{c}: \mathrm{Kn}=0.1)
$$

This reveals that two main circulations are formed. The main and the larger recirculation cell on the left, occupy a region between the left vertical wall and the front face of the step and recirculates clockwise. The second and weaker recirculation cell on the right is formed over the forward step that is counter-clockwise. By increasing the Grashof number, circulation has been stronger because the buoyancy forces have been stronger.

The streamline patterns are not significantly affected by the Knudsen number. However, the value of circulation strength decreases with an increase in Knudsen number. 
2.3. Effect of Grashof number, mean nanoparticle diameter, $R$ parameter and nanoparticle volume fraction on the Nusselt number

Figure (5-a) shows the variation of the Nusselt number vs. Grashof number. The Nusselt number increases with the increase of the Grashof number, as expected.

Figure (5-b) shows the average Nusselt number computed with different mean nanoparticle diameters for various Grashof numbers. By decreasing the mean diameters of nanoparticles from 200 to $5 \mathrm{~nm}$, the heat nmsfer with different Grashof numbers increases $28.9 \%$ and $20.7 \%$ for $\mathrm{Gr}=10^{2}$ and $\mathrm{Gr}=10^{2}$ aespectively.

Figure (5-c) shows the average Nusselt number computed with different $\mathrm{R}$ parameter for various Grashof numbers. By increasit traR parameter from 0.001to 0.008 , the Nusselt number increases $20.1 \%$ and $15.1 \%$ for $\mathrm{Gr}=10^{5}$, $\mathrm{rd} \mathrm{Gr}=10^{5}$, respectively.

Figure (5-d) shows the average Nusselt number computed with different nanoparticle volume fraction for various Grashof numbers. By increasing the mean nanoparticle volume fraction from 0.01to 0.05 , the Nusselt number increases $20.3 \%$ and $12.5 \%$ for $\mathrm{Gr}=10^{2}$ and $\mathrm{Gr}=10^{5}$, respectively.

a

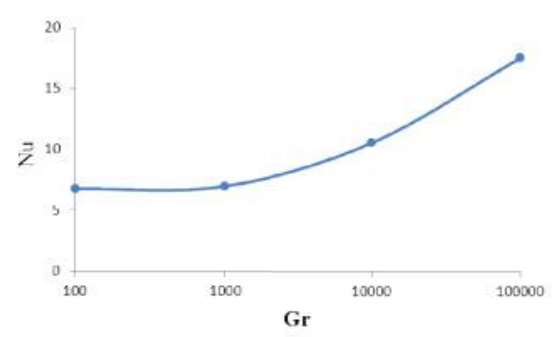

$\mathrm{c}$

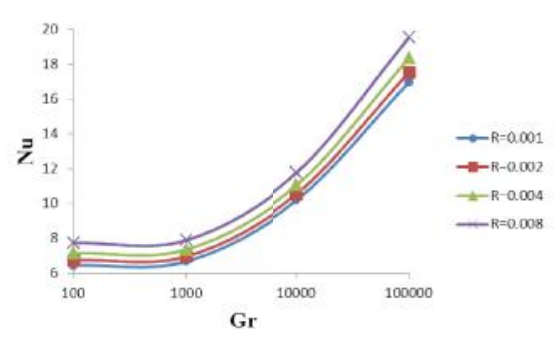

b

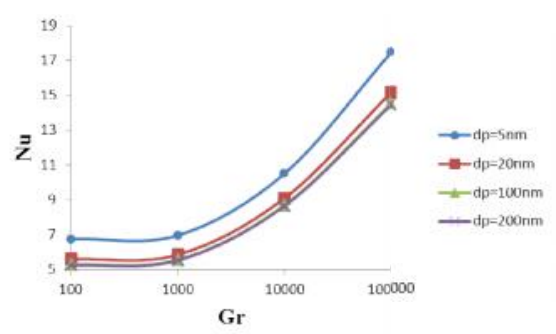

d

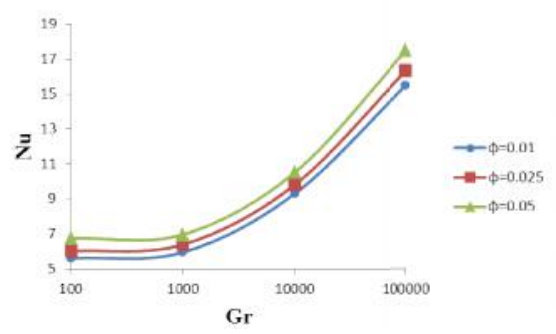

Fig.5. Variations of average Nusselt numbers with different parameters

\subsection{Effect of the Knudsen number on the Nusselt number}


Figure (6) shows the variation of the Nusselt number with the Knudsen number at different values of the Grashof number. It is seen that the Nusselt number decreases as the Knudsen number increases. Due to the temperature jump boundary condition, the bulk temperature of slip flow is lower than the temperature of continuumflow. The temperature jump is equivalent to a thermalcontact resistance between the wall and the fluid, while the slip velocityacts to decrease the thermal contact resistance. The velocityslip and the temperature jump bring about opposite effects on thetemperature difference between the fluid and the wall; the velocityslip tends to decrease the temperature difference between the fluid and the wall, while the temperature jump tends to increase it. Yu and Ameel[29] investigated slip flow heat transferin rectangular microchannels . They proposed that heat transfercould be increased or decreased compared to continuum flow conditionsdepending on B and Knudsen number, where B is definedas:

$$
\mathrm{B}=\frac{\frac{\left(2-\sigma_{\mathrm{T}}\right) 2 \mathrm{y}}{\sigma_{\mathrm{T}}(\mathrm{y}+1) \operatorname{Pr}}}{\frac{2-\sigma}{\sigma}}
$$

Actually, only when B and Knudsen number are very smalldoes it appear possible for the effects of velocity slip to win outover the opposite effects of the temperature jump and thus make aNusselt number greater than the continuum flow value. In otherwords, when the tangential momentum accommodation coefficientis significantly smaller than thermal accommodation coefficient,the Nusselt number will be greater than the continuum flowvalue. However, for practical engineering applications, it is extremelydifficult to realize and heat transfer is always reducedwhen slip flow occurs. In this study,by increasing the Knudsen number from 0.001to 0.1, the Nusseltnumber decreases $18.6 \%$ and $2.8 \%$ for $\mathrm{Gr}=10^{2}$ and $\mathrm{Gr}=10^{5}$, respectively.

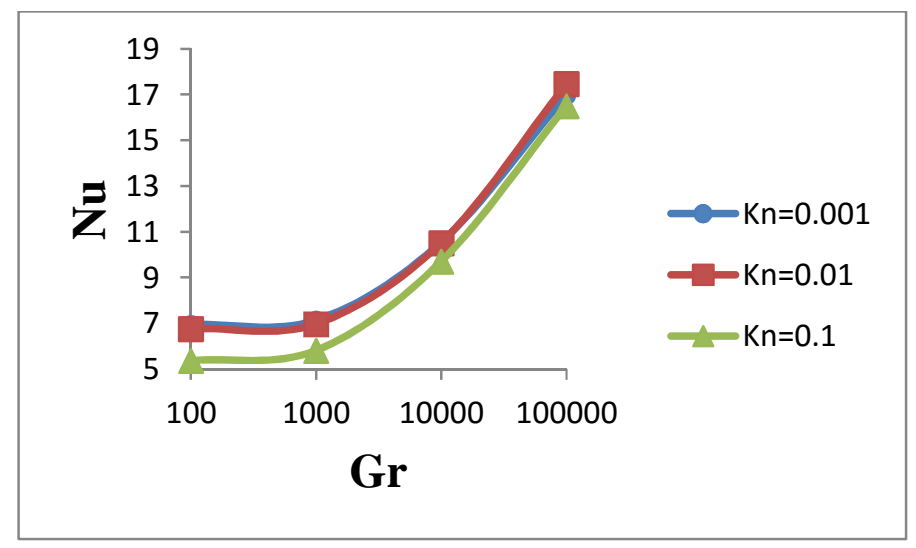

Fig.6. Variations of average Nusselt numbers with Knudsen number 


\section{EXPERIMENTAL}

This study investigates the flow field and heat transfer in a $\Gamma$ shaped micro-channel that is filled with $\mathrm{Al}_{2} \mathrm{O}_{3}$-watre nanofluid. Figure (7) shows the schematic of the model with its boundary conditions adopted in this study. The step is considered as isothermal at higher temperature than two vertical isothermal walls, the top and bottom walls are adiabatic.

Mathematical equations describing the physical model are based on the following assumptions:

(I) the thermo physical properties are constant except for the density in the buoyancy force (Boussinesq's hypothesis); (II) the fluid phase and nanoparticles are in a thermal equilibrium state; (III) nanoparticles are spherical; (IV) the nanofluid in the cavity is Newtonian, incompressible, and laminar; and $(\mathrm{V})$ radiation heat transfer between the sides of the cavity is negligible when compared with the other mode of heat transfer.

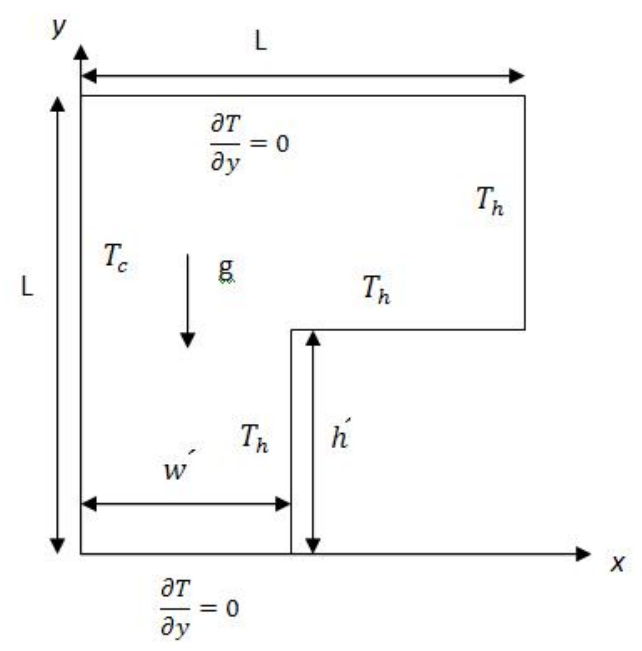

Fig.7. Schematic of the model and boundary condition

The equations of fluid motion in the Cartesian coordinate system are written as following (in dimensionless form):

Continuity equation

$$
\frac{\partial \mathrm{U}}{\partial \mathrm{X}}+\frac{\partial \mathrm{V}}{\partial \mathrm{Y}}=0
$$

$\mathrm{x}$ - momentum equation:

$$
\frac{\partial U}{\partial \xi}+U \frac{\partial U}{\partial X}+V \frac{\partial U}{\partial Y}=\frac{-\rho_{f}}{\rho_{n f}} \frac{\partial P}{\partial X}+\frac{\mu_{\text {eff }} P r}{\rho_{n f} V_{f}}\left(\frac{\partial^{2} U}{\partial X^{2}}+\frac{\partial^{2} U}{\partial Y^{2}}\right)
$$

y- momentum equation: 


$$
\frac{\partial \mathrm{V}}{\partial \xi}+\mathrm{U} \frac{\partial \mathrm{V}}{\partial \mathrm{X}}+\mathrm{V} \frac{\partial \mathrm{V}}{\partial \mathrm{Y}}=\frac{-\rho_{\mathrm{f}}}{\rho_{\mathrm{nf}}} \frac{\partial \mathrm{P}}{\partial \mathrm{Y}}+\frac{\mu_{\mathrm{eff}} \mathrm{Pr}}{\rho_{\mathrm{nf} \mathrm{V}_{\mathrm{f}}}}\left(\frac{\partial^{2} \mathrm{~V}}{\partial \mathrm{X}^{2}}+\frac{\partial^{2} \mathrm{~V}}{\partial \mathrm{Y}^{2}}\right)+\frac{(1-\Phi) \rho_{\mathrm{f}} \bar{\beta}_{\mathrm{f}}+\Phi \rho_{\mathrm{s}} \bar{\beta}_{\mathrm{s}}}{\rho_{\mathrm{nf}} \bar{\beta}_{\mathrm{f}}} \operatorname{Pr}^{2} \mathrm{Gr} \theta
$$

Energy equation:

$$
\frac{\partial \theta}{\partial \xi}+\mathrm{U} \frac{\partial \theta}{\partial \mathrm{x}}+\mathrm{V} \frac{\partial \theta}{\partial \mathrm{Y}}=\frac{\alpha_{\mathrm{nf}}}{\alpha_{\mathrm{f}}}\left(\frac{\partial^{2} \theta}{\partial \mathrm{X}^{2}}+\frac{\partial^{2} \theta}{\partial \mathrm{Y}^{2}}\right)
$$

The dimensionless form of equations obtained by using the following parameters:

$$
X=\frac{x}{L}, Y=\frac{y}{L}, U=\frac{u L}{\alpha_{f}}, V=\frac{v L}{\alpha_{f}}, \xi=\frac{\alpha_{f} t}{L^{2}}, P=\frac{p_{L}^{2}}{\rho_{f} \alpha_{f}}, \theta=\frac{T-T_{c}}{T_{h}-T_{c}}
$$

The main parameters in calculating the rate of heat transfer of nanofluids are important: Heat Capacity, Viscosity and Thermal conductivity which may be quite different from pure fluid.

The effective physical properties of the nanofluid in the above equations are:

$$
\mu_{\mathrm{eff}}=\mu_{\mathrm{f}}(1+2.5 \Phi)\left[1+\eta\left(\frac{\mathrm{d}_{\mathrm{p}}}{\mathrm{L}}\right)^{-2 \varepsilon} \Phi^{\frac{2}{3}}(\varepsilon+1)\right]
$$

This well-validated model is presented by Jang et al. (2007) [28] for a fluid containing a dilute suspension of small rigid spherical particles and it accounts for the slip mechanism in nanofluids. The empirical constant e and $\mathrm{g}$ are -0.25 and 280 for $\mathrm{Al}_{2} \mathrm{O}_{3}$, respectively.

Density:

There is a consensus among researchers in using the Law of mixtures in Calculation the density of nanofluids. This relationship is as follow:

$$
\rho_{\mathrm{nf}}=(1-\Phi) \rho_{\mathrm{f}}+\Phi \rho_{\mathrm{s}}
$$

Heat capacitance:

$$
\left(\rho c_{\mathrm{p}}\right)_{\mathrm{nf}}=(1-\Phi)\left(\rho \mathrm{c}_{\mathrm{p}}\right)_{\mathrm{f}}+\Phi\left(\rho \mathrm{c}_{\mathrm{p}}\right)_{\mathrm{s}}
$$

Thermal diffusivity:

$$
\alpha_{\mathrm{nf}}=\frac{\mathrm{k}_{\mathrm{nf}}}{\left(\rho c_{\mathrm{p}}\right)_{\mathrm{nf}}}
$$

Dimensionless stagnant thermal conductivity:

Research has shown that the thermal conductivity of the nanofluid is the function of both the base fluid thermal conductivity and thenanoparticles, also the volumetric density of surface area and the shape of nanoparticles and distribution volume fraction. This model, introduced by Hamilton and Crosser (H-C model, 1962) [31], considers the nanoparticles in the liquid as stationary.

$$
\frac{\mathrm{k}_{\text {stationary }}}{\mathrm{k}_{\mathrm{f}}}=\frac{\mathrm{k}_{\mathrm{s}}+2 \mathrm{k}_{\mathrm{f}}-2 \Phi\left(\mathrm{k}_{\mathrm{f}}-\mathrm{k}_{\mathrm{s}}\right)}{\mathrm{k}_{\mathrm{s}}+2 \mathrm{k}_{\mathrm{f}}+\Phi\left(\mathrm{k}_{\mathrm{f}}-\mathrm{k}_{\mathrm{s}}\right)}
$$




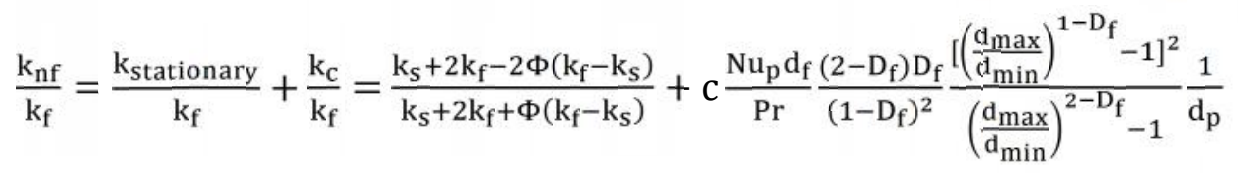

Total dimensionless thermal conductivity of nanofluids:

This model was proposed by $\mathrm{Xu}$ et al. [27] and it has been chosen in this study to describe the thermal conductivity of nanofluids. The first term is the $\mathrm{H}-\mathrm{C}$ model and the second term is the thermal conductivity based on heat convection due to Brownian motion. $\mathrm{C}$ is an empirical constant, which is relevant to the thermal boundary layer and dependent on different fluids (e.g. c $=85$ for the deionized water and $\mathrm{c}=280$ for ethylene glycol) but independent of the type of nanoparticles. $\mathrm{Nu}_{\mathrm{p}}$ is the Nusselt number for liquid flowing around a spherical particle and equals to two for a single particle in this work. The fluid molecular diameter $\mathrm{d}_{\mathrm{f}}$ is taken as $4.5 \times$ $10^{-10} \mathrm{~m}$ for water in present study. The fractal dimension $D_{\mathrm{f}}$ is determined by:

$D_{\mathrm{f}}=2-\frac{\operatorname{Ln} \Phi}{\operatorname{Ln}\left(\frac{\mathrm{d}, \min }{\mathrm{d}_{\mathrm{p}, \max }}\right)}$

Where $d_{p, \max }$ and $d_{p, \text { min }}$ are the maximum and minimum diameters of nanoparticles, respectively. With the given measured ratio of $d_{p, \min } / d_{p, \max }$, the minimum and maximum diameters of nanoparticles can be obtained with mean nanoparticle diameter $d_{p}$ from the statistical property of fractal media.

$d_{p, \text { max }}=d_{p} \frac{D_{f}-1}{D_{f}}\left(\frac{d_{p, \min }}{d_{p, \text { max }}}\right)^{-1}$

$d_{p, \min }=d_{p} \frac{D_{f}-1}{D_{f}}$

Boundary condition

In the slip flow region, due to the rarefaction effect, the flow will slip and the temperature will jump at the wall. The expression used for velocity is [32]:

$\mathrm{U}_{\mathrm{f}}-\mathrm{U}_{\mathrm{V}}=\lambda \frac{2-\sigma}{\sigma} \frac{\partial \mathrm{U}}{\partial \mathrm{n}}$

Where $\lambda$ is the molecular mean free path. The constant $\lambda$ denotes tangential momentum accommodation coefficient, which is usually between 0.87 and 1 [33]. Although the nature of the tangential momentum accommodation coefficients is still an active research problem, almost all evidence indicates that for most interactions the coefficients are approximately 1.0.

And the expression used for temperature is [34]: 
$\theta_{\mathrm{f}}-\theta_{\mathrm{V}}=\frac{2-\sigma_{\mathrm{t}}}{\sigma_{\mathrm{t}}} \frac{2 \gamma}{\gamma+1} \frac{\lambda}{\operatorname{Pr}} \frac{\partial}{\partial \mathrm{n}}$

In which $\sigma_{\mathrm{t}}$ represents a thermal accommodation coefficient measuring the extent to which the energies of molecules impinging on a surface are affected by contact with the surface. $\sigma_{\mathrm{t}}$ is usually between 0.32 and 1 [31] and close to unity for typical engineering surfaces. $\gamma$ denote the specific heat ratio.

Nusselt number

For nanofluids local Nusselt number $(\mathrm{Nu})$ is defined as:

$\mathrm{Nu}=-\frac{\mathrm{k}_{\mathrm{nf}}}{\mathrm{k}_{\mathrm{f}}} \frac{\partial \theta}{\partial \mathrm{n}}$

Where, $\mathrm{n}$ denoted the normal direction on a plan. The local Nusselt number at the heat wall plane $\mathrm{Nu}_{\mathrm{x}}, \mathrm{Nu}_{\mathrm{y}}$ are defined as follows:

$\mathrm{Nu}_{\mathrm{x}}=-\frac{\mathrm{k}_{\mathrm{nf}}}{\mathrm{k}_{\mathrm{f}}} \frac{\partial \theta}{\partial \mathrm{Y}}$

$M u_{y}=-\frac{k_{n f}}{k_{f}} \frac{\partial \theta}{\partial x}$

Herefore, the average Nusselt number is calculated as follow:

ikt $=\frac{1}{\mathrm{~h}+(1-\mathrm{w})}\left(\int_{0}^{\mathrm{h}} \mathrm{Nu}_{\mathrm{y}}+\int_{\mathrm{w}}^{1} \mathrm{Nu}_{\mathrm{x}}\right)$

3. Cell centered finite-volume scheme for space discretization

By integrating from conservation Eqs. (1)-(4) over a control volume , which is bounded by $\partial$, and applying the Gauss divergence theorem, one gets:

$$
\begin{aligned}
& \iint_{\Omega} \frac{1}{\beta} \frac{\partial \mathrm{P}}{\partial \zeta} \mathrm{ds}=-\oint_{\partial \Omega}(\mathrm{UdY}-\mathrm{VdX}) \\
& \iint_{\Omega} \frac{\partial \mathrm{U}}{\partial \zeta} \mathrm{ds}=-\frac{\rho_{\mathrm{f}}}{\rho_{\mathrm{nf}}} \oint_{\partial \Omega} \mathrm{PdY}-\int_{\partial \Omega}\left(\mathrm{U}^{2} \mathrm{dY}-\mathrm{UVdX}\right)+\frac{\mu_{\mathrm{eff}} \mathrm{Pr}}{\rho_{\mathrm{ff}} \mathrm{V}_{\mathrm{f}}} \int_{\partial \Omega}\left(\frac{\partial \mathrm{U}}{\partial \mathrm{X}} \mathrm{d} \mathrm{Y}-\frac{\partial \mathrm{U}}{\partial \mathrm{Y}} \mathrm{dX}\right) \\
& \iint_{\Omega} \frac{\partial \mathrm{V}}{\partial \zeta} \mathrm{ds}=\frac{-\rho_{\mathrm{f}}}{\rho_{\mathrm{nf}}} \oint_{\partial \Omega} \mathrm{PdX}-\int_{\partial \Omega}\left(\mathrm{UVd} \mathrm{d}-\mathrm{V}^{2} \mathrm{dX}\right)+\frac{\mu_{\mathrm{eff}} \mathrm{Pr}}{\rho_{\mathrm{nf} \mathrm{f}}} \int_{\partial \Omega}\left(\frac{\partial \mathrm{V}}{\partial \mathrm{X}} \mathrm{dY}-\frac{\partial \mathrm{V}}{\partial \mathrm{Y}} \mathrm{dX}\right) \\
& +\frac{(1-\Phi) \rho_{\mathrm{f}} \bar{\beta}_{\mathrm{f}}+\Phi \rho_{\mathrm{s}} \bar{\beta}_{\mathrm{s}}}{\rho_{\mathrm{nf}} \bar{\beta}_{\mathrm{f}}} \operatorname{Pr}^{2} \mathrm{Gr} \theta \iint_{\partial \Omega} \mathrm{ds} \\
& \iint_{\Omega} \frac{\partial \theta}{\partial \zeta} \mathrm{ds}=\frac{\alpha_{\mathrm{nf}}}{\alpha_{\mathrm{f}}} \int_{\partial \Omega}\left(\frac{\partial \theta}{\partial \mathrm{X}} \mathrm{dY}-\frac{\partial \theta}{\partial \mathrm{Y}} \mathrm{dX}\right)-\int_{\partial \Omega}(\mathrm{U} \theta \mathrm{dY}-\mathrm{V} \theta \mathrm{dX})
\end{aligned}
$$

Therefore, these equations can be arranged in a suitable vector form as follows:

$\frac{\partial}{\partial \zeta} \iint_{\Omega} \mathrm{Qds}=-\oint_{\partial \Omega}(\mathrm{FdY}-\mathrm{GdX})+\oint_{\partial \Omega}(\mathrm{RdY}-\mathrm{WdX})+\iint_{\partial \Omega} \mathrm{Zd} s$ 


$$
\begin{gathered}
\mathrm{Q}=\left(\begin{array}{c}
\mathrm{P} \\
\mathrm{U} \\
\mathrm{V} \\
\theta
\end{array}\right), \quad \mathrm{F}=\left(\begin{array}{c}
\beta \mathrm{U} \\
\mathrm{U}^{2}+\mathrm{P} \\
\mathrm{UV} \\
\mathrm{U} \theta
\end{array}\right), \quad \mathrm{G}=\left(\begin{array}{c}
\beta \mathrm{V} \\
\mathrm{UV} \\
\mathrm{V}^{2}+\mathrm{P} \\
\mathrm{V} \theta
\end{array}\right), \quad \mathrm{R}=\left(\begin{array}{c}
0 \\
\frac{\mu_{\mathrm{eff}} \mathrm{Pr}}{\rho_{\mathrm{nf}} \mathrm{V}_{\mathrm{f}}} \frac{\partial \mathrm{U}}{\partial \mathrm{X}} \\
\frac{\mu_{\mathrm{eff}} \mathrm{Pr}}{\rho_{\mathrm{nf}}} \frac{\partial \mathrm{V}}{\partial \mathrm{X}} \\
\frac{\alpha_{\mathrm{nf}}}{\alpha_{\mathrm{f}}} \frac{\partial \theta}{\partial X}
\end{array}\right) \\
\mathrm{W}=\left(\begin{array}{c}
0 \\
\frac{\mu_{\mathrm{eff}} \mathrm{Pr}}{\rho_{\mathrm{nfvf}}} \frac{\partial U}{\partial \mathrm{Y}} \\
\frac{\mu_{\mathrm{eff}} \mathrm{Pr}}{\rho_{\mathrm{nf}} \mathrm{v}_{\mathrm{f}}} \frac{\partial \mathrm{V}}{\partial \mathrm{Y}} \\
\frac{\alpha_{\mathrm{nf}} \frac{\partial \theta}{\alpha_{\mathrm{f}}}}{\partial \mathrm{Y}}
\end{array}\right), \mathrm{Z}=\left(\begin{array}{c}
0 \\
\frac{(1-\Phi) \rho_{\mathrm{f}} \bar{\beta}_{\mathrm{f}}+\Phi \rho_{s} \bar{\beta}_{\mathrm{s}}}{\rho_{\mathrm{nf}} \bar{\beta}_{\mathrm{f}}} \operatorname{Pr}^{2} \mathrm{Gr} \theta \\
0
\end{array}\right)
\end{gathered}
$$

The computational domain is divided into quadrilateral cells (see figure 2) and a system of ordinary differential equations is obtained by applying Eq. (23) to each cell separately.

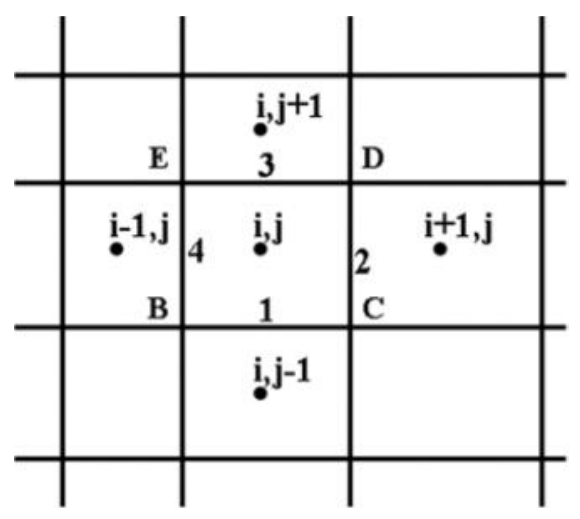

Fig.8.The cell for discretization and averaging

In order to find the convective terms in the boundaries of the cell $(i, j)$, the custom flux averaging scheme is chosen. In this scheme each quantity such as $(U \theta)_{A E}$ (i.e. the value of $u \theta$ on the boundary denoted by number 1 as shown in Fig. 2), is evaluated as the average of the cells on either side of the face (i.e. $\left.(\mathrm{U} \theta)_{\mathrm{AE}}=0.5\left[(\mathrm{U} \theta)_{\mathrm{i}, \mathrm{j}}+(\mathrm{U} \theta)_{\mathrm{i}, \mathrm{j}-1}\right]\right)$.

The flux averaging is applied here for the viscous and conduction terms. In this new method, we need to know the first derivatives such as $\left(\frac{\partial \theta}{\partial \mathrm{Y}}\right)_{\mathrm{i}, \mathrm{j}}$ and so on (at the cell's center). These functions are obtained from the Gauss divergence theorem. For example: $\left(\frac{\partial \theta}{\partial \mathrm{Y}}\right)_{\mathrm{i}, \mathrm{j}}=-\frac{1}{\mathrm{~s}_{\mathrm{i}, \mathrm{j}}} \oint_{\partial \Omega} \theta \mathrm{dx}$ Therefore, these derivatives are saved for all cells as the functions that can be used in the next time step. Thus each quantity for example $\left(\frac{\partial \theta}{\partial \mathrm{Y}}\right)_{\mathrm{AE}}$ is found such as: $\left(\frac{\partial \theta}{\partial \mathrm{Y}}\right)_{\mathrm{AE}}=0.5\left[\left(\frac{\partial \theta}{\partial \mathrm{Y}}\right)_{\mathrm{i}, \mathrm{j}}+\right.$ 
$\left(\frac{\partial \theta}{\partial \mathrm{Y}}\right)_{\mathrm{i}, \mathrm{j}-1}$ ].After discretizing Eq. (21) around cell $(\mathrm{i}, \mathrm{j})$ and to consider the cell centered assumption for variables:

$\mathrm{S}_{\mathrm{i}, \mathrm{j}} \frac{\partial \mathrm{Q}_{\mathrm{i}, \mathrm{j}}}{\partial \zeta}=-\sum_{\mathrm{k}=1}^{4}\left(\mathrm{~F}_{\mathrm{i}, \mathrm{j}} \Delta \mathrm{Y}-\mathrm{G}_{\mathrm{i}, \mathrm{j}} \Delta \mathrm{X}\right)_{\mathrm{K}}+\sum_{\mathrm{k}=1}^{4}\left(\mathrm{R}_{\mathrm{i}, \mathrm{j}} \Delta \mathrm{Y}-\mathrm{W}_{\mathrm{i}, \mathrm{j}} \Delta \mathrm{X}\right)_{\mathrm{K}}+\mathrm{S}_{\mathrm{i}, \mathrm{j}} \mathrm{Z}_{\mathrm{i}, \mathrm{j}}$

where, $S_{i, j}$ is the area of $(i, j)$ the cell.

\section{CONCLUSIONS}

In this paper, a computational code was developed using a cell-centered finite-volume methodfor solving theincompressible natural convection nanofluidflow within a $\Gamma$-shaped micro-channel in the slip flow region, including the effects of velocity slip and temperature jump at the wall, which are the main characteristics of flow in the slip flow region.An explicit fourth-orderRunge-Kutta integration algorithm was applied to find thesteady state condition. Also an artificial compressibility techniquewas applied to couple thecontinuity to the momentum equations. In this work, the Grashof number, the mean diameter of nanoparticles, R parameter, and the Knudsen number, were limited as: $10^{2} \leq G r \leq 10^{5}, 5 \mathrm{~nm} \leq d_{p} \leq 200 \mathrm{~nm}, 0.001 \leq R \leq 0.008,0 \leq$ $K n \leq 10^{-1}$ respectively.Results show that decreasing the mean diameters of nanoparticles, increasing $\mathrm{R}$ parameter, and increasing the mean nanoparticle volume fraction, increase the Nusselt number. Alsoincreasing the Knudsen number decreases the Nusselt number, in other words the slip flow Nusselt numbers are lower than those forcontinuum flow and decrease with an increase in Knudsen number.

\section{REFERENCES}

[1] H. Masuda, A. Ebata, K. Teramae, N. Hishinuma, Alteration of thermal conductivity and viscosity of liquid by dispersing ultra-fine particles (dispersions of - $\mathrm{Al} 2 \mathrm{O} 3, \mathrm{SiO} 2$, and $\mathrm{TiO} 2$ ultra-fine particles).NetsuBussei (Japanese) 4, 1993, 227-233.

[2] C.H. Li, G.P. Peterson, Experimental investigation of temperature and volume fraction variations on the effective thermal conductivity of nanoparticle suspensions, J. Appl. Phys., 2006, 99, 084314.

[3] Ali Ijam, R. Saidur, Nanofluid as a coolant for electronic devices (cooling of electronic devices), J. Applied Thermal Engineering, 2012, 32, 76-82. 
[4] Tun-Ping Teng, Yi-Hsuan Hung, Tun-ChienTeng, Huai-En Mo, How-Gao Hsu, The effect of alumina/water nanofluid particle size on thermal conductivity, J. Applied Thermal Engineering, 2010, 30, 2213-2218.

[5] DorinLelea, CatalinNisulescu, The micro-tube heat transfer and fluid flow of water based A12O3 nanofluid with viscous dissipation, International Communications in Heat and Mass Transfer, 2011, 38, (6), 704-710.

[6] Ch.Ch. Choa, Ch. Chen, Ch. K. Chen, Natural convection heat transfer and entropy generation in wavy-wall enclosure containing water-based nanofluid, International Journal of Heat and Mass Transfer, 2013, 61, 749-758.

[7] A. H. Mahmoudia, I. Pop, M. Shahia, F. Talebia, MHD natural convection and entropy generation in a trapezoidal enclosure using $\mathrm{Cu}$-water nanofluid, Computers \& Fluids, 2013, $72,46-62$.

[8] Arie M, Shooshtari A, Dessiatoun S, Al-Hajri E, Ohadi M., Numerical modeling and thermal optimization of a single-phase flow manifold-micro-channel plate heat exchanger. International Journal of Heat and Mass Transfer, 2015, 81, 478-89.

[9] P. FariñasAlvariño, J.M. SáizJabardo, A. Arce, M.I. Lamas Galdo, A numerical investigation of laminar flow of a water/alumina nanofluid, International Journal of Heat and Mass Transfer, 2013, 423-432.

[10] Ebrahimi A, Roohi E, Kheradmand S., Numerical Study of Liquid Flow and Heat Transfer in Rectangular Micro-channel with Longitudinal Vortex Generators. Applied Thermal Engineering, 2014, 78, 576-83.

[11] Yue Y, Mohammadian SK, Zhang Y, Analysis of performances of a manifold micro-channel heat sink with nanofluids. International Journal of Thermal Sciences, 2015, 89, 305- 13.

[12] Hedayati F, Domairry G., Effects of nanoparticle migration and asymmetric heating on mixed convection of TiO 2-H 2 O nanofluid inside a vertical micro-channel . Powder Technology, 2015, 272, 250-59.

[13] X. D. Wang, B. An, J. L.Xu, Optimal geometric structure for nanofluid-cooled microchannel heat sink under various constraint conditions, Energy Conversion and Management, 2013, 65, 528-538. 
[14] S. M. HosseiniHashemi, S. A. Fazeli, H. Shokouhmand, Fully developed non-Darcian forced convection slip-flow in a micro-annulus filled with a porous medium: Analytical solution, Energy Conversion and Management, 2011, 52(2), 1054-1060.

[15] QinboHea, Shuangfeng Wang, ShequanZeng, ZhaozhiZheng, Experimental investigation on photothermal properties of nanofluids for direct absorption solar thermal energy systems, Energy Conversion and Management, 2013, 73, 150-157.

[16] Massimo Corcione, Empirical correlating equations for predicting the effective thermal conductivity and dynamic viscosity of nanofluids, Energy Conversion and Management, 2011, 52(1), 789-793.

[17]Zhu X.W, Fu Y.H, Zhao J.Q, Zhu L., Three-dimensional numerical study of the laminar flow and heat transfer in a wavy-finned heat sink filled with A12O3/ethylene glycol-water nanofluid. Numerical Heat Transfer Part A (2015): Applications: 1-14.

[18] A. Jameson, W. Schmidt, E. Turkel, Numerical simulation of the Euler equations by finite volume method using Runge-Kutta time stepping schemes, AIAA Paper 81-1259, in: 14th Fluid and Plasma Dynamics Conference, Palo Alto, California; June 1981.

[19] A. Jameson, D. Mavriplis, Finite volume solution of the two-dimensional Euler equations on a regular triangular mesh, AIAA 24, 1986, 611-618.

[20]V. Esfahanian, P. Akbarzadeh, The Jameson's numerical method for solving the incompressible viscous and inviscid flows by means of artificial compressibility and preconditioning method, Applied Mathematics and Computation, 2008, 206(2), 651-661.

[21] S.V. Patankar, Numerical Heat Transfer and Fluid Flow, McGraw-Hill, Washington, 1980.

[22] A.J. Chorin, A numerical method for solving incompressible viscous flow problems, J. Comput. Phys., 1967, 12-26.

[23] A.J. Chorin, A numerical method for solving incompressible viscous flow problems, J. Comput. Phys., 1997, 112, 118-125.

[24]P.A. Madsen, H.A. Schäffer, A discussion of artificial compressibility, Coastal Eng., 2006, 53, 93-98.

[25]F. Bassi, A. Crivellini, D.A. Pietro, S. Rebay, An artificial compressibility flux for the discontinuous Galerkin solution of the incompressible Navier-Stokes equations, J. Comput. Phys., 2006, 218, 794-815. 
[26]B.M. Ziapour, R. Dehnavi, Finite-volume method for solving the entropy generation due to air natural convection in $\Gamma$-shaped enclosure with circular corners, Math. Comput.Modelling, 2011, 54, 1286-1299.

[27]J. Xu, B. Yu, M. Zou, P. Xu, A new model for heat conduction of nanofluids based on fractal distributions of nanoparticles, J. Phys. D 39, 2006, 4486-4490.

[28] S.P. Jang, J.H. Lee, K.S. Hwang, S.U.S. Choi, Particle concentration and tube size dependence of viscosities of Al2O3-water nanofluids flowing through micro- and mini tubes. Appl. Phys. Lett. 91, 2007, 243112.

[29] ResamDehnavi, AbdollahRezvani, Numerical investigation of natural convection heat transfer of nanofluids in a C shaped cavity, Superlattices and Microstructures, 2012, 52, 312325.

[30] Yu, S. P., Ameel, T. A. Slip Flow Heat Transfer in Rectangular micro-channels ,” Int. J. Heat Mass Transfer, 2001, 44(22), 4225-4234.

[31] R.L. Hamilton, O.K. Crosser, Thermal conductivity of heterogeneous two component systems.Indus. Eng. Chem. Fund. 1962, 1, 187-191.

[32]Zhang T, Jia L, Yang L, Jaluria Y, Effect of viscous heating on heat transfer performance in micro-channel slip flow region, International Journal of Heat and Mass Transfer, 2010, 53 4927-4934.

[33]Rohsenow, W. M., and Choi, H. Y. Heat, Mass, and Momentum Trans-fer, Prentice-Hall, Englewood Cliffs, NJ, Chap. 11, 1961.

[34] Shojaeian M, Dibaji R, Three-dimensional numerical simulation of the slip flow through triangular micro-channels, International Communications in Heat and Mass Transfer, 2010, 37 324-329.

\section{How to cite this article:}

Rouzbahani $\mathrm{F}$ and Dehnavi R. Numerical investigation of natural convection heat transfer of nano-fluids in a micro-channel. J. Fundam. Appl. Sci., 2016, 8(2S), 448-466. 